\title{
HE 0141-3932: A bright QSO with an unusual emission line spectrum and associated absorption $\star, \star \star$
}

\author{
D. Reimers ${ }^{1}$, E. Janknecht ${ }^{1}$, C. Fechner ${ }^{1}$, I. I. Agafonova ${ }^{2}$, S. A. Levshakov ${ }^{2}$, and S. Lopez ${ }^{3}$ \\ ${ }^{1}$ Hamburger Sternwarte, Universität Hamburg, Gojenbergsweg 112, 21029 Hamburg, Germany \\ 2 Department of Theoretical Astrophysics, Ioffe Physico-Technical Institute, 194021 St. Petersburg, Russia \\ e-mail: lev@astro.ioffe.rssi.ru \\ ${ }^{3}$ Departamento de Astronomia, Universidad de Chile, Casilla 36-D, Santiago, Chile
}

Received 13 July 2004 / Accepted 19 January 2005

\begin{abstract}
HE 0141-3932 $\left(z_{\mathrm{em}}=1.80\right)$ is a bright blue radio-quite quasar with an unusually weak Ly $\alpha$ emission line. Large redshift differences $(\Delta z=0.05)$ are observed between high ionization and low ionization emission lines. Absorption systems identified at $z_{\text {abs }}=1.78,1.71$, and 1.68 show mild oversolar metallicities $\left(Z \approx 1-2 Z_{\odot}\right)$ and can be attributed to the associated gas clouds ejected from the circumnuclear region. The joint analysis of the emission and absorption lines leads to the conclusion that this quasar is seen almost pole-on. Its apparent luminosity may be Doppler boosted by $\sim 10$ times. The absorbing gas shows a high abundance of $\mathrm{Fe}, \mathrm{Mg}$ and $\mathrm{Al}([\mathrm{Fe}, \mathrm{Mg}, \mathrm{Al} / \mathrm{C}] \simeq 0.15 \pm 0.10)$ along with underabundance of $\mathrm{N}([\mathrm{N} / \mathrm{C}] \leq-0.5)$. This abundance pattern is at variance with current chemical evolution models of QSOs predicting $[\mathrm{N} / \mathrm{C}] \gtrsim 0$ and $[\mathrm{Fe} / \mathrm{C}]<0$ at $Z \sim Z_{\odot}$.
\end{abstract}

Key words. cosmology: observations - line: formation - line: profiles - galaxies: abundances - quasars: absorption lines quasars: individual: HE 0141-3932

\section{Introduction}

In the course of a high-resolution study of the Ly $\alpha$ forest at intermediate redshifts $(1.5 \leq z \leq 2)$ in bright quasars from the Hamburg/ESO Survey with the UV-visual echelle spectrograph (UVES) at the VLT, the discovery spectrum of the QSO HE 0141-3932 $(z \simeq 1.8, B=16.2$, Wisotzki et al. 2000) attracted our attention by two facts: it appeared to have no or only very weak $\operatorname{Ly} \alpha$ emission along with clearly recognizable Mg II, and it showed several absorption line systems at $z_{\text {abs }}$ ranging from 1.78 to 1.68 with very complex and strong metal profiles which allow us to suggest that these systems may originate in the ejected gas.

Since the identification spectrum of HE 0141-3932 was of only moderate quality, we took further low-resolution spectra with EFOSC 2 with the ESO $3.6 \mathrm{~m}$ telescope to improve the redshift measurement and we found a third peculiarity, namely large redshift differences $(\Delta z \sim 0.05)$ between high ionization and low ionization emission lines.

Emission line spectra similar to that of HE 0141-3932 are observed in some high redshift BL Lacertae (BL Lac) objects

^ Based on observations obtained at the VLT Kueyen telescope and 3.6 m ESO telescope, Chile, the ESO programs Nos. 67.A-0280(A) and 72.D-0174A, respectively.

$\star \star$ Appendix $\mathrm{A}$ is only available in electronic form at http://www. edpsciences.org
(Urry \& Padovani 1995). However, HE 0141-3932 is a radioquiet quasar, i.e. not a blazar. A few other blue radio-quiet quasars are known with apparently missing or weak Ly $\alpha$ emission but clearly present metal lines: PG $1407+265\left(z_{\mathrm{em}}=0.94\right.$, McDowell et al. 1995), Tol 1037-2703 $\left(z_{\mathrm{em}}=2.20\right.$, Srianand \& Petitjean 2001), PHL $1811\left(z_{\mathrm{em}}=0.192\right.$, Leighly et al. 2004), and several cases from SDSS (Fan et al. 1999, 2003). The nature of their peculiar emission line spectra is not clear.

In particular, Leighly et al. (2004) suggest a high accretion rate which powers the UV emission from an optically thick accretion disk, while suppressing the formation of a hot corona. However, recent radio observations of PG 1407+265 on the milliarcsecond scale revealed a relativistic jet of moderate power beamed toward us (Blundell et al. 2003). This means that the quasar is seen almost pole-on and its emission line spectrum may be diluted by continuum radiation both from the jet and the accretion disk.

In the present paper we analyze both the emission and absorption line spectra of HE 0141-3932. We expect that this joint consideration may shed some light on the unusual properties of this quasar.

The paper is organized as follows: observations are described in Sect. 2, the emission lines are studied in Sect. 3, the analysis of the $z_{\mathrm{abs}} \approx z_{\mathrm{em}}$ absorbers is given in Sect. 4 , the results are discussed and summarized in Sect. 5. 



Fig. 1. EFOSC 2 spectra of HE 0141-3932. The spectral resolutions are indicated in the panels. All identified emission features are labeled. The parameters of the prominent emission lines are listed in Table 2.

Table 1. Log of spectroscopic observations of HE 0141-3932.

\begin{tabular}{llcll}
\hline \hline Instrument & \multicolumn{1}{c}{$\begin{array}{c}\lambda \lambda \\
(\AA)\end{array}$} & $\begin{array}{c}\text { Exposure } \\
\text { time }(\mathrm{h})\end{array}$ & Date & $\begin{array}{l}\text { Resolution } \\
\left(\mathrm{km} \mathrm{s}^{-1}\right)\end{array}$ \\
\hline UVES & $3053-3872$ & 7 & & 7.3 \\
& $4700-5800$ & 7 & July + & 7.3 \\
& $5800-6800$ & 7 & August & 7.8 \\
UVES & $3761-4982$ & 4 & 2001 & 7.3 \\
& $6650-8550$ & 4 & & 7.8 \\
\multirow{2}{*}{ EFOSC 2 } & $8600-10400$ & 4 & & 7.8 \\
& $3270-5240$ & 0.25 & Oct. 2 & $420(6 \AA)$ \\
& $3380-7520$ & 0.25 & 2003 & $700(13 \AA)$ \\
\hline
\end{tabular}

\section{Observations}

HE 0141-3932 was observed with the UVES at the $8 \mathrm{~m}$ ESO VLT on Paranal over 7 nights in July/August 2001. Eleven individual exposures with integration times of 60 min were made using the dichroic mode in standard settings (Table 1). With a slit width of $1^{\prime \prime}$, a resolution of $41000\left(7.3 \mathrm{~km} \mathrm{~s}^{-1}\right)$ in the blue and $38000\left(7.9 \mathrm{~km} \mathrm{~s}^{-1}\right)$ in the red is achieved.

The data reduction was performed at the Quality Control Group in Garching using the UVES pipeline Data Reduction Software (Ballester et al. 2000), the vacuum-barycentric corrected spectra were co-added. The resulting signal-to-noise ratio, $S / N$, is typically 75 .

Since both the $\operatorname{Ly} \alpha$ and the $\mathrm{Mg}$ II emission line ranges are covered only by UVES spectra, we performed an absolute flux calibration using an appropriate master response curve and the respective airmasses during the observations. The procedure is described on the ESO web page www.eso.org/observing/ $\mathrm{dfo/quality/UVES/qc/response.html.}$

Further spectra were taken with EFOSC 2 at the ESO $3.6 \mathrm{~m}$ telescope on October 2, 2003, to improve our knowledge about the redshifts of the emission lines. Details are given in Table 1. Wavelength and flux calibration was performed according to standard procedures. The resulting spectra are shown in Fig. 1. Notice that due to the rapidly decreasing sensitivity below $3500 \AA$, the EFOSC 2 spectra only partially cover the wavelength range expected for the Ly $\alpha$ emission line.

\section{The emission line spectrum}

The combination of EFOSC 2 spectra with roughly flux calibrated UVES spectra allows us to estimate redshifts and equivalent widths of all emission lines between $\operatorname{Ly} \alpha$ and Mg II $\lambda 2800 \AA$ Aquivalent widths $(E W)$ and redshifts were measured by fitting Gaussian profiles to the data. Table 2 presents the results. The Ly $\alpha E W$ is relatively uncertain since in the calibrated UVES data the continuum is difficult to determine and the blue wing of the line is incomplete (see Figs. 1 and 2).

The identification of the emission line at $\sim 3400 \AA$ is ambiguous: it could be either $\operatorname{Ly} \alpha$ at $z=1.80$ or $\mathrm{N} \mathrm{V}$ at $z=1.75$ or blend of both lines. There are two arguments in favor of the $\mathrm{N}$ V identification: it has a width comparable to C IV and its redshift - if identified as $\mathrm{NV}$ - is equal to that of the C IV, while in QSOs with significant redshift differences between high ionization lines (C IV) and low ionization lines ( $\mathrm{Mg} \mathrm{II}, \mathrm{H}_{\alpha}$ ), the Ly $\alpha$ line typically is found at the redshift of the high ionization 
Table 2. Estimated equivalent widths, redshifts and relative velocities for emission lines of HE 0141-3932.

\begin{tabular}{lccccl}
\hline \hline Ion & Instrument & $E W_{\text {rest }}, \AA$ & $z_{\text {obs }}$ & $v, \mathrm{~km} \mathrm{~s}^{-1}$ & Comment \\
\hline H I 1215 & UVES & $16 \pm 7$ & $1.80_{-0.02}^{+0.01}$ & $0_{-210}^{+1070}$ & Asymmetric \\
and/or N v 1239 & & & $1.75 \pm 0.01$ & $-5350 \pm 1070$ & \\
Si IV 1398+O IV] 1402 & EFOSC 2 & $11 \pm 1$ & $1.76 \pm 0.01$ & $-4280 \pm 1070$ & - \\
C IV 1549 & EFOSC 2 & $14 \pm 1$ & $1.75 \pm 0.01$ & $-5350 \pm 1070$ & Asymmetric \\
Al III 1858 + & EFOSC 2 & $13 \pm 1$ & $1.79 \pm 0.01$ & $-1070 \pm 1070$ & - \\
C III] 1909 + Si III] 1892 & & & $1.79 \pm 0.01$ & $-1070 \pm 1070$ & Blend, fractions of components unknown \\
Fe III 2075 & EFOSC 2 & $5 \pm 1$ & $1.80 \pm 0.01$ & $0 \pm 1070$ & - \\
Mg II 2800 & UVES & $16 \pm 1$ & $1.795 \pm 0.005$ & $-535 \pm 535$ & Blended with Fe II emission \\
\hline
\end{tabular}

Zero velocity corresponds to $z=1.80$. Only statistical errors are indicated.

BLR emission lines (Gaskell 1982; Espey et al. 1989). On the other hand, the emission line at $3400 \AA$ is quite strong for $\mathrm{N} \mathrm{V}$, although there are quasars known with $\mathrm{N} \mathrm{V}$ strength comparable to that of C IV (Hall et al. 2004; Baldwin et al. 2003b). Even if this line would be entirely due to emission of neutral hydrogen, we can conclude that Ly $\alpha$ in HE 0141-3932 is unusually weak.

The second anomaly of this quasar is the large velocity difference between low ionization lines ( $\mathrm{Mg}$ II, C III], Al III, etc.) and high ionization lines (C IV, Si IV) of roughly $5000 \mathrm{~km} \mathrm{~s}^{-1}$; it is among the most extreme cases like PG $1407+265$ (McDowell et al. 1995, $\Delta v_{\mathrm{MgII}-\mathrm{CIV}} \simeq 5000 \mathrm{~km} \mathrm{~s}^{-1}$ ) and Q 1309-056 (Espey et al. 1989, $\Delta v_{\mathrm{Mg} \mathrm{II}-\mathrm{C} \mathrm{IV}} \simeq 4000 \mathrm{~km} \mathrm{~s}^{-1}$ ).

In Fig. 2 we display the emission line profiles on a velocity scale relative to $z=1.80$. The redshift of the low ionization lines of $z=1.80$ appears to be roughly the systemic redshift, since the UVES spectrum shows that the Ly $\alpha$ forest starts at $z=1.8086$.

While the velocity shift in the emission lines is extremely large, how unusual are the emission line intensities? This can be best seen by a comparison with the histogram of equivalent widths of the LBQS-quasars, a well selected sample of QSO given by Francis (1993). The Ly $\alpha, \mathrm{C} \mathrm{IV}, \mathrm{C}$ III] and Mg II equivalent widths are among the bottom $2 \%, 1 \%, 13 \%$ and $1.5 \%$, respectively, of the Francis (1993) distribution. At the same time, the Al III 1856/64 and Fe III 2075 lines appear unusually strong. In order to investigate the physical properties of gas which could produce such an unusual emission line spectrum we compared the observed equivalent widths of HE 0141-3932 with the compilation of quasar BLR rest-wavelength emission line equivalent widths given as functions of column density, incident ionizing spectrum, and metal abundance of the emitting gas clouds (Korista et al. 1997). While Ly $\alpha$, Mg II and C III] have roughly the same velocity (redshift) and could originate in the same volume, we were unable to find a parameter combination which met the constraints on $\operatorname{Ly} \alpha$ and $\mathrm{Mg}$ II line strengths simultaneously - the theoretical Ly $\alpha$ is always much too strong for a parameter combination that matches the $\mathrm{Mg}$ II equivalent width. The $\mathrm{C}$ IV/Ly $\alpha E W$ ratio can be met assuming high gas densities $[\log n(\mathrm{H}) \geq 12]$, but the discrepant redshifts exclude formation in the same location. A mean BLR parameter set

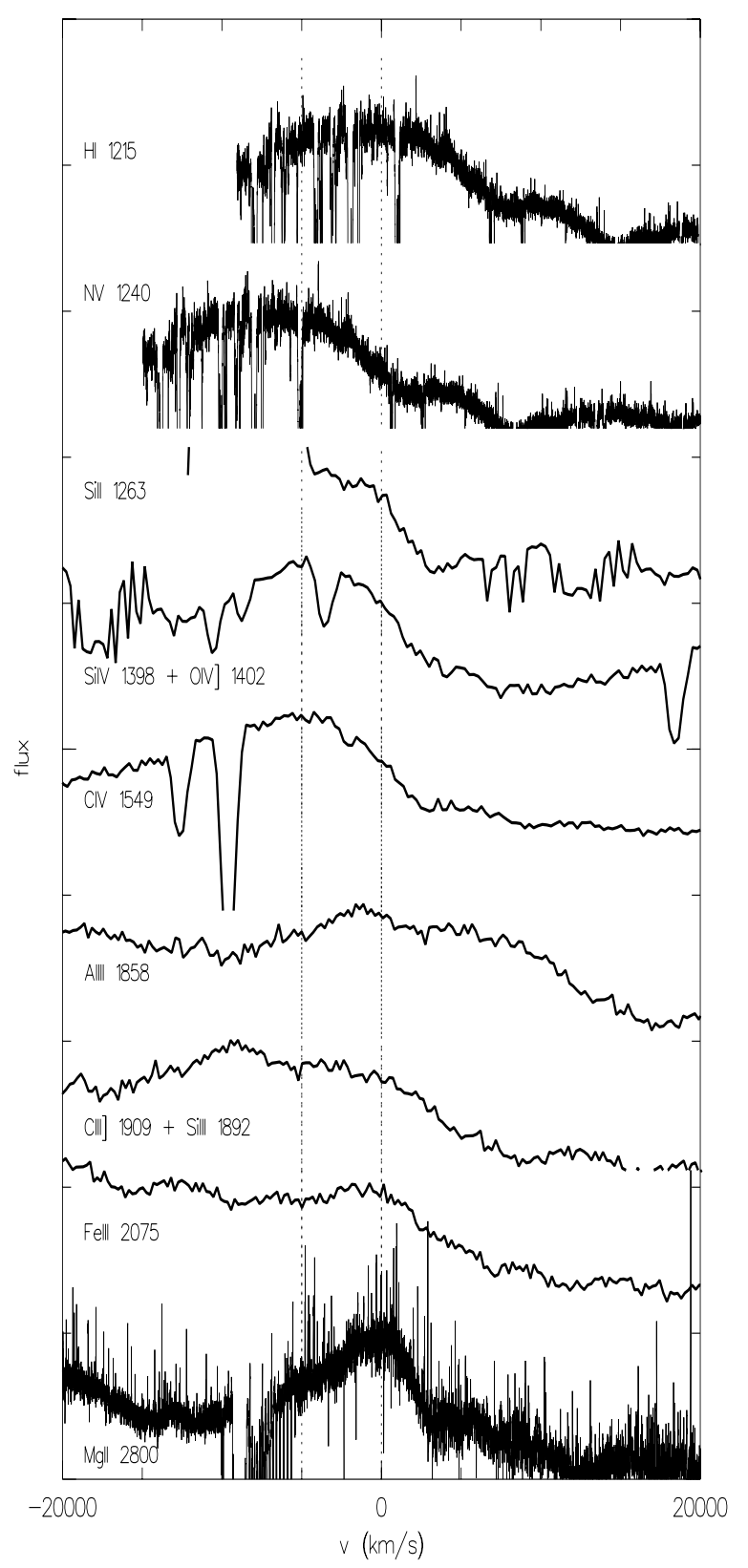

Fig. 2. Emission lines of HE 0141-3932 (spectra from UVES and EFOSC 2). $v=0 \mathrm{~km} \mathrm{~s}^{-1}$ corresponds to $z=1.80$. For orientation, at $v=0 \mathrm{~km} \mathrm{~s}^{-1}$ and $v=-5000 \mathrm{~km} \mathrm{~s}^{-1}$ dashed lines are plotted. 
appears to be unable to reproduce the measured $E W$ s of emission lines in HE 0141-3932.

\section{The absorption systems}

\subsection{Calculation procedure}

In order to estimate the physical parameters of the absorption systems we used the Monte Carlo Inversion (MCI) method. Detailed description of the MCI is given in Levshakov et al. (2000, 2002, 2003a). Here we outline briefly its basics needed to understand the results presented.

The MCI is based on the assumption that all lines observed in the absorption system are formed in a continuous absorbing gas slab of thickness $L$ where the gas density, $n_{\mathrm{H}}(x)$, and velocity, $v(x)$, fluctuate from point to point giving rise to complex profiles (here $x$ is the space coordinate along the line of sight).

We also assume that within the absorber the metal abundances are constant, the gas is optically thin for the ionizing UV radiation, and the gas is in thermal and ionization equilibrium. The intensity and the spectral shape of the background ionizing radiation are treated as external parameters.

The radial velocity $v(x)$ and gas density $n_{\mathrm{H}}(x)$ are considered as two continuous random functions which are represented by their sampled values at equally spaced intervals $\Delta x$. The computational procedure is based on adaptive simulated annealing. The fractional ionizations of different elements at each space coordinate $x, \Upsilon_{\text {ion }}[U(x)]$, are computed with the photoionization code CLOUDY (Ferland 1997).

The following physical parameters are directly estimated by the MCI procedure: the mean ionization parameter $U_{0}$, the total hydrogen column density $N_{\mathrm{H}}$, the line-of-sight velocity dispersion, $\sigma_{\mathrm{v}}$, and density dispersion, $\sigma_{\mathrm{y}}$, of the bulk material $\left[y \equiv n_{\mathrm{H}}(x) / n_{0}\right]$, and the chemical abundances $Z_{\mathrm{a}}$ of all elements involved in the analysis. With these parameters we can further calculate the column densities for different species $N_{\mathrm{a}}$, and the mean kinetic temperature $T_{\text {kin }}$.

In general, the uncertainties on the fitting parameters $U_{0}$, $N_{\mathrm{H}}, \sigma_{v}, \sigma_{y}$, and $Z_{a}$ are about $15 \%-20 \%$ (for data with $S / N \gtrsim 30$ ) and the errors of the estimated column densities are less than $10 \%$. However, in individual absorption systems the accuracy of the recovered values can be lower for different reasons like partial blending of the line profiles, saturation, or absence of lines of subsequent ionic transitions.

The MCI can be supplemented with an additional procedure aimed at restoring the shape of the background ionizing spectrum. A formal description of this procedure is given in the Appendix. Its accuracy depends significantly on the number of unsaturated lines of the subsequent ionic transitions of different elements (e.g. Si II/Si III/Si IV, C II/C III/C IV) available for the analysis. The absorber at $z_{\mathrm{abs}}=1.7817$ (described below) reveals enough such lines and we use them to estimate the spectral shape of the background $U V$ radiation in the range $E>1$ Ryd.

All calculations are carried out with the laboratory wavelengths and oscillator strengths taken from Morton (2003). Solar photospheric abundance for carbon is taken from
Allende Prieto et al. (2002), for silicon, nitrogen and iron from Holweger (2001).

\subsection{Absorption system at $z_{a b s}=1.7817$}

Many unsaturated lines of different ionic transitions along with a single saturated hydrogen line Ly $\alpha$ are detected in this system (Fig. 3) separated by $2000 \mathrm{~km} \mathrm{~s}^{-1}$ from the central source. The blue wing of Ly $\alpha$ is partially blended by the adjacent hydrogen line $\left[N(\mathrm{HI}) \sim 10^{14} \mathrm{~cm}^{-2}\right]$ from an intervening absorber (only a weak C IV doublet is detected in this system). C III $\lambda 977$ is beyond the wavelength coverage and Si III $\lambda 1206$ is blended, probably with $\operatorname{Ly} \alpha$ absorption from the $z_{\text {abs }}=1.7606$.

We started the analysis assuming standard ionizing backgrounds such as a power law $f_{v} \propto v^{-\alpha}$ (with different indexes $\alpha=1.0-1.8$ ), the mean intergalactic spectrum (at $z=1.8$ ) of Haardt \& Madau (1996), and the AGN-type spectrum of Mathews \& Ferland (1987, hereafter MF).

None of these spectra was consistent with the observed intensities of the CII, CIV, Si II and Si IV lines: all trials underproduced $\mathrm{C}$ II/C IV and overproduced $\mathrm{Si}$ II/Si IV, but the MF spectrum provided the lowest $\chi^{2}$. All spectra gave the mean ionization parameter in the range of $-2.75 \lesssim \log U_{0} \lesssim-2.5$. In spite of the saturation, the neutral hydrogen column density can be estimated with a sufficiently high accuracy $(\sim 20 \%)$ since the velocity dispersion of gas is determined by numerous metal lines detected in this system (the procedure to restore partly blended profiles is described in Sect. 3 in Levshakov et al. 2003a). All runs with different model spectra showed a rather high metallicity - slighty above solar value, and an almost solar ratio of $\mathrm{Si} / \mathrm{C}$.

Taking into account this preliminary information we can assume that the UV spectrum to be found should maximize the product of ratios $\mathrm{CII} / \mathrm{C}$ IV and $\mathrm{Si}$ IV/Si II in the range of $U_{0} \sim 0.002-0.003$ for solar metal content and solar element abundances. The spectral shape of the MF spectrum can be taken as a first approximation (for more details see Appendix).

Applying the adjustment procedure as described in Appendix, we estimated a new shape of the ionizing spectrum which ensured a self-consistent description of all lines observed in the $z_{\mathrm{abs}}=1.7817$ system. This spectrum is shown in Fig. 4 by the dashed line, whereas the initial MF spectrum is the solid line. As seen in Fig. 4, the restored spectrum is softer in the range 2 Ryd $<E<6.5$ Ryd but much harder above 6.5 Ryd. It shows a break at the He II ionization edge with the amplitude $J_{v}(\mathrm{~A}) / J_{v}(\mathrm{~B}) \simeq 3$ corresponding to the optical depth $\tau_{\mathrm{c}}(\mathrm{He}$ II $) \simeq 1$. Physical parameters estimated with this modified ionizing background are listed in Table 3, Col. 2, and the corresponding synthetic profiles are plotted in Fig. 3 by the smooth curves.

\subsection{Absorption system at $z_{a b s}=1.7103$}

This absorption system spans the velocity range of $500 \mathrm{~km} \mathrm{~s}^{-1}$ and is located in velocity space at a distance of $9600 \mathrm{~km} \mathrm{~s}^{-1}$ from the QSO. It reveals many lines of different ionic transitions (Fig. 5). The structure of the Ly $\alpha, \mathrm{C}$ IV, Si IV and N V 


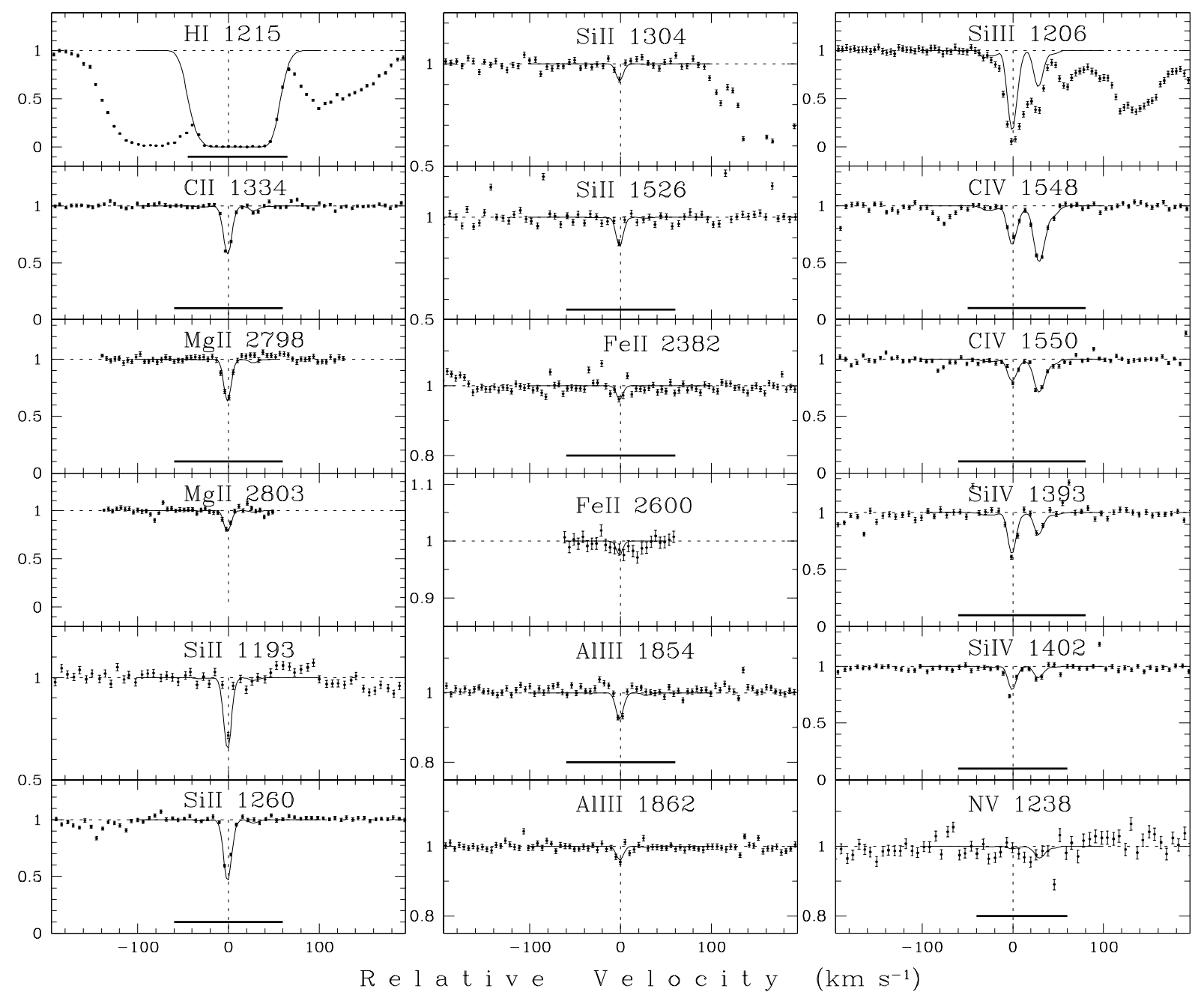

Fig. 3. Hydrogen and metal absorption lines associated with the $z_{\text {abs }}=1.7817$ system toward HE 0141-3932 (normalized intensities are shown by dots with $1 \sigma$ error bars). The zero radial velocity is fixed at $z=1.7817$. Smooth curves are the synthetic spectra convolved with the corresponding point-spread spectrograph function and computed with the physical parameters listed in Table 3, Col. 2. Bold horizontal lines mark pixels included in the optimization procedure. The synthetic profiles of unmarked absorption features were calculated in a second round using the velocity $v(x)$ and gas density $n_{\mathrm{H}}(x)$ distributions already obtained in the optimization procedure.

profiles indicates that the system may be divided into two subsystems: one at $-200 \mathrm{~km} \mathrm{~s}^{-1}<v<180 \mathrm{~km} \mathrm{~s}^{-1}(A)$ and another at $180 \mathrm{~km} \mathrm{~s}^{-1}<v<320 \mathrm{~km} \mathrm{~s}^{-1}(B)$.

The subsystem $B$ has an unsaturated hydrogen line and hence allows us to estimate the hydrogen column density with a sufficiently high accuracy. The physical parameters computed with the UV background deduced for the $z_{\mathrm{abs}}=1.7817$ system are given in Table 3, Col. 4 . The obtained extremely high metallicity - almost $9 Z_{\odot}-$ is striking. Since silicon lines Si III $\lambda 1206$ and Si IV $\lambda 1393,1402$ are very weak and the element ratios $([\mathrm{Si} / \mathrm{C}],[\mathrm{N} / \mathrm{C}])$ not known a priori, the mean ionization parameter $U_{0} \simeq 0.04$ represents a lower limit consistent with the observed intensities of the C IV lines and the absence of the C II $\lambda 1334$ absorption. Formally this subsystem could be fitted with any higher ionization parameter producing even higher metallicity and higher $[\mathrm{Si} / \mathrm{C}]$ and $[\mathrm{C} / \mathrm{N}]$ ratios.

To confirm the high metallicity in this subsystem we repeated calculations assuming other ionizing backgrounds power laws with $\alpha$ ranging between 1.0 and 1.8 and the
MF spectrum. These trials also delivered metallicity of about 10 solar.

In principle, such high metal enrichment of the circumnuclear gas is predicted in some models of chemical evolution of QSO/host galaxies (Hamann \& Ferland 1999). However, another explanation of our results is that the absorbing gas is not in equilibrium with the ionizing background, e.g., is still cooling and recombining. In this case high metallicities can be caused by a longer recombination time of hydrogen as compared to C IV and N V (e.g., Osterbrock 1989). The ionization parameter $U$ and, hence, the total hydrogen column density $N_{\mathrm{H}} \propto 1 / \Upsilon_{\mathrm{HI}}(U)$ is determined by the observed line intensities of different ions. Since hydrogen is ionized higher than it would be in the ionization equilibrium with the same parameter $U$, the total hydrogen becomes underestimated leading to an artificially high metallicity. We may expect that the adjacent subsystem $A$ (centered at $v=0 \mathrm{~km} \mathrm{~s}^{-1}$ ) would help to clarify the physical conditions in the $z_{\mathrm{abs}}=1.7103$ absorber.

The subsystem $A$ shows complex profiles of low ionization lines C II, Mg II, Si II, and Fe II along with Al III, Si III, Si IV, 


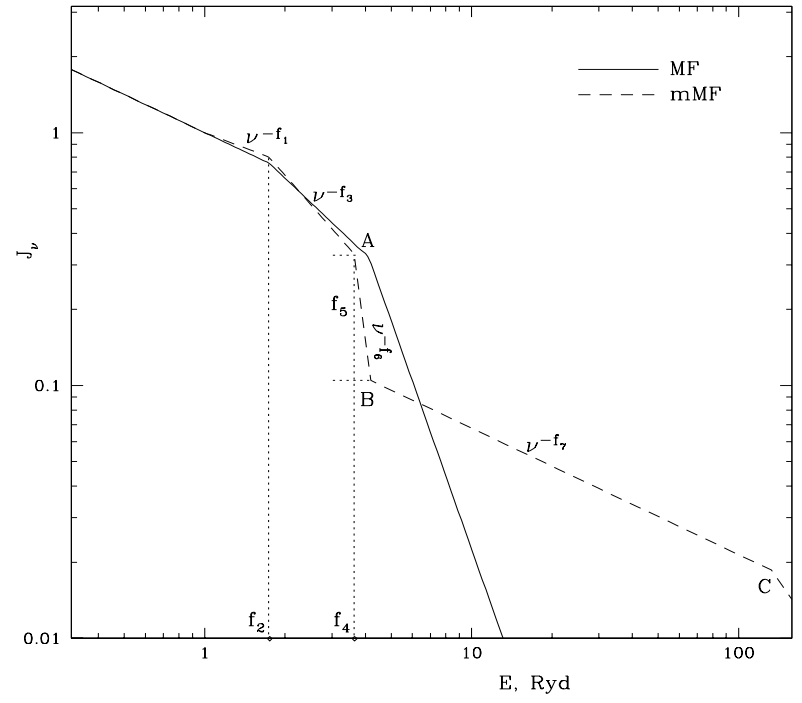

Fig. 4. Spectral shape of a typical AGN ionizing continuum from Mathews \& Ferland (1987) shown by the solid line and its modification (dashed line) estimated for the $z_{\text {abs }}=1.7817$ system. The spectra are normalized so that $J_{v}(h v=1 \mathrm{Ryd})=1$. Factors $f_{i}$ are defined in the Appendix.

N V and saturated C IV (see Fig. 5). The computations had been carried out with the ionizing background from the $z_{\text {abs }}=1.7817$ system. The presence of the subsequent ionic transitions guarantees the accuracy of the mean ionization parameter of $\sim 10 \%$ (for a given ionizing background).

The assumption of constant metallicity inside the absorber turned out to be inconsistent with the observed blue wing of Ly $\alpha$ and the absence of any metal absorption in the range $-200 \mathrm{~km} \mathrm{~s}^{-1}<v<-100 \mathrm{~km} \mathrm{~s}^{-1}$. A single available hydrogen line does not allow us to conclude whether the blue wing is blended or there is indeed a gradient of metal content. The former possibility looks more probable since there is a nonzero flux in the Ly $\alpha$ profile at $-110 \mathrm{~km} \mathrm{~s}^{-1}<v<-90 \mathrm{~km} \mathrm{~s}^{-1}$ with the mean intensity $0.018 \pm 0.005$ (marked by the arrow in Fig. 5). The constant metallicity throughout this sub-system seems to be appropriate since very steep blue wings of several ions (Si III, Si IV, C IV) do not indicate a progressive dilution. Thus, the calculations were carried out with the assumption of constant metallicity. The neutral hydrogen column density and the shape of the blue wing of the synthetic Ly $\alpha$ shown in Fig. 5 were calculated with the velocity and density fields estimated from the observed metal line profiles (for details see Sect. 4.3 in Levshakov et al. 2003a). The obtained physical parameters are given in Table 3, Col. 3. We also tried other ionizing backgrounds (different power laws and the MF spectrum), but none of them was consistent with the observed relative intensities within the C II/C IV and Si II/Si III/Si IV profiles.

In spite of all uncertainties intrinsic to this subsystem several parameters were steadly reproduced in all runs. These are (1) slightly undersolar nitrogen abundance, $[\mathrm{N} / \mathrm{C}]=-0.1 \pm 0.1$; (2) significantly lower ratios of $\mathrm{Si} / \mathrm{C}$ and $\mathrm{Al} / \mathrm{C}$ as compared to their solar values, $[\mathrm{Si} / \mathrm{C}]=-0.3 \pm 0.1,[\mathrm{Al} / \mathrm{C}]=-0.3 \pm 0.1$; and (3) extremely high overabundance of iron $[\mathrm{Fe} / \mathrm{H}] \gtrsim 1.5$. The extremely high overabundance of iron as well as underabundances of silicon and aluminium may be caused by non-equilibrium ionization. When an absorbing gas comes to equilibrium through cooling and recombining its ionization parameter can be overstated due to different cooling and recombination times of the observed ions stemming from density fluctuations (so-called "hot photoionization"). A higher density gas has shorter cooling and recombination times and hence comes faster to equilibrium. Thus, the sub-system $A$ may be described as consisting of dense gas clumps that are already close to equilibrium (seen in low ionization absorptions) and ambient rarefied gas still far from equilibrium (responsible for most of C IV and $\mathrm{N} \mathrm{V}$ absorptions). The equilibrium ionization parameter should be lower, probably ranging between 0.001 and 0.003 . With this $U_{0}$, only a mild overabundance of iron, $[\mathrm{Fe} / \mathrm{Si}] \sim 0.2-0.3$, would be enough to describe the observed intensity of the Fe II lines.

Thus, we estimate a metallicity of $\gtrsim 9 Z_{\odot}$ in the higher ionized subsystem $B$ and $\sim 2 Z_{\odot}$ in the lower ionized subsystem $A$ (both metallicities are referred to silicon lines). This strong metallicity difference is another argument in favor of non-equilibrium ionization in sub-system $B$. It probably has lower gas density than in $A$ which leads to longer cooling and recombination times. We suggest that some time ago the whole absorbing complex $(A+B)$ was either exposed to a much more intense radiation or shock-heated up to the temperatures when collisional ionization becomes significant (Klein et al. 1994, 2003; Levshakov et al. 2004).

\subsection{Absorption system at $z_{a b s}=1.6838$}

This system separated by $\sim 12450 \mathrm{~km} \mathrm{~s}^{-1}$ from the QSO shows a partially saturated Ly $\alpha$ and many metal lines with complex profiles (Fig. 6). Si II $\lambda 1260,1193,1190$ and NV $\lambda 1242$ are blended with Ly $\alpha$ forest absorptions, and at the position of Si II $\lambda 1526$ a clear continuum window is seen.

The apparent structure of the $\operatorname{Ly} \alpha$ profile suggests that this system can be divided into two subsystems: one at $-250 \mathrm{~km} \mathrm{~s}^{-1}<v<-180 \mathrm{~km} \mathrm{~s}^{-1}(A)$ and another at $-180 \mathrm{~km} \mathrm{~s}^{-1}<v<150 \mathrm{~km} \mathrm{~s}^{-1}(B)$ which were analyzed separately. Calculations were carried out using both the ionizing spectrum restored for the $z_{\mathrm{abs}}=1.7817$ system and several power law spectra. Physical parameters listed in Table 3, Cols. 5, 6 correspond to the $z_{\text {abs }}=1.7817$ background. The red wing of the synthetic profile of $\operatorname{Ly} \alpha$ in the subsystem $B$ is calculated simultaneously with metal lines assuming a constant metallicity inside the absorber. The ionization parameter given in Table 3 for the subsystem $A$ is determined from the observed intensity of C IV and the noise level at the expected position of the $\mathrm{C}$ II $\lambda 1334$ line and should be considered as a lower limit.

The almost solar ratio of $[\mathrm{Si} / \mathrm{C}]=0.06 \pm 0.1$ and a strong underabundance of nitrogen $[\mathrm{N} / \mathrm{C}]<-0.5$ obtained for both subsystems indicate that photoionization is probably close to equilibrium. However, slightly higher metallicity in the subsystem $A$ and the remaining flux $0.04 \pm 0.01$ at the shallow bottom of Ly $\alpha$ may indicate that hydrogen has not yet reached its equilibrium with the ionizing background (see sub-system $B$ in the foregoing section). Although the absolute values of the 
Table 3. Physical parameters of the $z_{\mathrm{abs}}=1.7817,1.7103$ and 1.6838 metal absorbers toward HE $0141-3932\left(z_{\mathrm{em}}=1.80\right)$ derived by the MCI procedure (limits are given at the $1 \sigma$ level).

\begin{tabular}{|c|c|c|c|c|c|}
\hline \multirow[b]{2}{*}{$\begin{array}{l}\text { Parameter } \\
(1)^{f}\end{array}$} & \multirow{2}{*}{$\begin{array}{c}z_{\mathrm{abs}}=1.7817 \\
\text { (2) }\end{array}$} & \multicolumn{2}{|c|}{$z_{\mathrm{abs}}=1.7103$} & \multicolumn{2}{|c|}{$z_{\mathrm{abs}}=1.6838$} \\
\hline & & 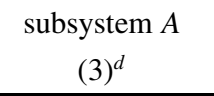 & $\begin{array}{l}\text { subsystem } B \\
(4)^{d}\end{array}$ & $\begin{array}{c}\text { subsystem } A \\
\text { (5) }\end{array}$ & $\begin{array}{c}\text { subsystem } B \\
\text { (6) }\end{array}$ \\
\hline$U_{0}$ & $3.1 \mathrm{E}-3^{a}$ & $9.0 \mathrm{E}-3$ & $3.8 \mathrm{E}-2$ & $\gtrsim 2.8 \mathrm{E}-2$ & $1.3 \mathrm{E}-2^{a}$ \\
\hline$N_{\mathrm{H}}, \mathrm{cm}^{-2}$ & $4.2 \mathrm{E} 17^{a}$ & 5.7E18 & $8.6 \mathrm{E} 17$ & $\gtrsim 3.2 \mathrm{E} 17$ & $2.5 \mathrm{E} 18$ \\
\hline$\sigma_{\mathrm{v}}, \mathrm{km} \mathrm{s}^{-1}$ & $20.8^{a}$ & 55.0 & 21.0 & 19.2 & $59.0^{a}$ \\
\hline$\sigma_{\mathrm{y}}$ & $0.63^{a}$ & 0.77 & 0.62 & 0.36 & $0.52^{a}$ \\
\hline$Z_{\mathrm{C}}$ & $3.4 \mathrm{E}-4^{a}$ & $1.1 \mathrm{E}-3$ & $2.1 \mathrm{E}-3$ & $<5.2 \mathrm{E}-4$ & $2.9 \mathrm{E}-4^{a}$ \\
\hline$Z_{N}$ & $<1.2 \mathrm{E}-4$ & $3.1 \mathrm{E}-4$ & $3.2 \mathrm{E}-4$ & $\lesssim 5.0 \mathrm{E}-5$ & $2.8 \mathrm{E}-5^{b}$ \\
\hline$Z_{\mathrm{Mg}}$ & $7.0 \mathrm{E}-5^{b}$ & $1.4 \mathrm{E}-4$ & $\ldots$ & $\ldots$ & $\ldots$ \\
\hline$Z_{\mathrm{Al}}$ & $7.0 \mathrm{E}-6^{b}$ & $6.2 \mathrm{E}-6$ & $\ldots$ & $\ldots$ & $\ldots$ \\
\hline$Z_{\mathrm{Si}}$ & $4.3 \mathrm{E}-5^{a}$ & $7.4 \mathrm{E}-5$ & $3.0 \mathrm{E}-4$ & $\lesssim 8.5 \mathrm{E}-5$ & $4.8 \mathrm{E}-5^{a}$ \\
\hline$Z_{\mathrm{Fe}}$ & $6.0 \mathrm{E}-5^{b}$ & $\sim 8.0 \mathrm{E}-4$ & $\ldots$ & $\ldots$ & $\ldots$ \\
\hline$\left[Z_{C}\right]$ & $0.14 \pm 0.08$ & 0.65 & 0.94 & $\lesssim 0.33$ & $0.08 \pm 0.10$ \\
\hline$\left[Z_{N}\right]$ & $<0.15$ & 0.56 & 0.58 & $\lesssim-0.3$ & $-0.48 \pm 0.15$ \\
\hline$\left[Z_{\mathrm{Mg}}\right]$ & $0.30 \pm 0.10$ & 0.6 & $\ldots$ & $\ldots$ & $\ldots$ \\
\hline$\left[Z_{\mathrm{Al}}\right]$ & $0.30 \pm 0.10$ & 0.3 & $\ldots$ & $\ldots$ & $\ldots$ \\
\hline$\left[Z_{\mathrm{Si}}\right]$ & $0.09 \pm 0.08$ & 0.33 & 0.94 & $<0.4$ & $0.14 \pm 0.10$ \\
\hline$\left[Z_{\mathrm{Fe}}\right]$ & $0.30 \pm 0.15$ & $\sim 1.5$ & $\ldots$ & $\ldots$ & $\ldots$ \\
\hline$N(\mathrm{H} \mathrm{I}), \mathrm{cm}^{-2}$ & $(2.6 \pm 0.5) \mathrm{E} 15$ & $1.0 \mathrm{E} 16^{e}$ & $(2.6 \pm 0.5) \mathrm{E} 14$ & $(1.1 \pm 0.2) \mathrm{E} 14$ & $2.5 \mathrm{E} 15^{e}$ \\
\hline$N(\mathrm{C}$ II $), \mathrm{cm}^{-2}$ & $(1.64 \pm 0.08) \mathrm{E} 13$ & $(1.5 \pm 0.2) \mathrm{E} 14$ & $\lesssim 1.0 \mathrm{E} 12$ & $\lesssim 1.6 \mathrm{E} 11$ & $(7.3 \pm 1.5) \mathrm{E} 12$ \\
\hline$N\left(\mathrm{C} \mathrm{II}^{*}\right), \mathrm{cm}^{-2}$ & $<5.0 \mathrm{E} 11$ & $<4.3 \mathrm{E} 12$ & $\ldots$ & $\ldots$ & $<1.5 \mathrm{E} 12$ \\
\hline$N(\mathrm{Mg} \mathrm{II}), \mathrm{cm}^{-2}$ & $(1.20 \pm 0.20) \mathrm{E} 11$ & $(5.3 \pm 1.5) \mathrm{E} 12$ & $\ldots$ & $\ldots$ & $\ldots$ \\
\hline$N(\mathrm{Si} \mathrm{II}), \mathrm{cm}^{-2}$ & $(2.8 \pm 0.3) \mathrm{E} 12$ & $(1.2 \pm 0.1) \mathrm{E} 13$ & $\ldots$ & $\cdots$ & $\ldots$ \\
\hline$N(\operatorname{Si~II}), \mathrm{cm}^{-2}$ & $<1.3 \mathrm{E} 11$ & $<2.0 \mathrm{E} 11$ & $\ldots$ & $\ldots$ & $\ldots$ \\
\hline$N(\mathrm{Fe} \mathrm{II}), \mathrm{cm}^{-2}$ & $(1.4 \pm 0.4) \mathrm{E} 11$ & $(5.5 \pm 2.0) \mathrm{E} 11$ & $\ldots$ & $\ldots$ & $\ldots$ \\
\hline$N(\mathrm{Al} \mathrm{III}), \mathrm{cm}^{-2}$ & $(3.6 \pm 1.0) \mathrm{E} 11$ & $(1.4 \pm 0.3) \mathrm{E} 12$ & $\ldots$ & $\ldots$ & $\cdots$ \\
\hline$N(\mathrm{Si}$ III $), \mathrm{cm}^{-2}$ & $7.5 \mathrm{E} 12^{c}$ & $(5.5 \pm 0.6) \mathrm{E} 13$ & $(2.3 \pm 1.2) \mathrm{E} 11$ & $\lesssim 8.5 \mathrm{E} 10$ & $(7.9 \pm 0.8) \mathrm{E} 12$ \\
\hline$N(\mathrm{C}$ IV $), \mathrm{cm}^{-2}$ & $(2.2 \pm 0.2) \mathrm{E} 13$ & $(1.7 \pm 0.2) \mathrm{E} 15$ & $(3.4 \pm 0.3) \mathrm{E} 14$ & $(3.4 \pm 0.2) \mathrm{E} 13$ & $(2.3 \pm 0.1) \mathrm{E} 14$ \\
\hline$N(\mathrm{Si}$ IV $), \mathrm{cm}^{-2}$ & $(4.5 \pm 0.4) \mathrm{E} 12$ & $(7.7 \pm 0.8) \mathrm{E} 13$ & $(1.6 \pm 0.5) \mathrm{E} 12$ & $<3.0 \mathrm{E} 11$ & $(1.2 \pm 0.1) \mathrm{E} 13$ \\
\hline$N(\mathrm{~N} \mathrm{v}), \mathrm{cm}^{-2}$ & $<1.4 \mathrm{E} 12$ & $(1.8 \pm 0.2) \mathrm{E} 14$ & $(7.7 \pm 0.8) \mathrm{E} 13$ & $(4.5 \pm 0.5) \mathrm{E} 12$ & $(1.3 \pm 0.1) \mathrm{E} 13$ \\
\hline$\langle T\rangle, \mathrm{K}$ & $0.8 \mathrm{E} 4$ & $1.2 \mathrm{E} 4$ & $1.3 \mathrm{E} 4$ & $1.8 \mathrm{E} 4$ & $1.6 \mathrm{E} 4$ \\
\hline
\end{tabular}

${ }^{a, b}$ Uncertainties: $^{a} \sim 15-20 \%$, and ${ }^{b} \sim 30 \%$.

${ }^{c}$ The value of $N(\mathrm{Si} \mathrm{III})$ is calculated on base of other lines.

${ }^{d}$ Physical parameters $\left(U_{0}, N_{\mathrm{H}}, \sigma_{\mathrm{v}}, \sigma_{\mathrm{y}}\right.$ and metallicities) are only illustrative since estimated for the photoionization equilibrium whereas the absorption system is in non-equilibrium.

${ }^{e}$ Estimated under the assumption of constant metallicity inside the absorber.

${ }^{f} Z_{\mathrm{X}}=N_{\mathrm{X}} / N_{\mathrm{H}} ;\left[Z_{\mathrm{X}}\right]=\log \left(N_{\mathrm{X}} / N_{\mathrm{H}}\right)-\log \left(N_{\mathrm{X}} / N_{\mathrm{H}}\right)_{\odot}$.

abundances are uncertain (they depend on the assumed background and the mean ionization parameter), the solar to oversolar carbon content, the ratio $[\mathrm{Si} / \mathrm{C}] \simeq 0$ and a significant underabundance of nitrogen are constantly reproduced in all trials.

\subsection{Absorption systems at $z_{a b s}=1.7365$ and 1.4978}

Other systems with unusually strong and complex C IV profiles are identified in the spectrum of HE 0141-3932. Unfortunately, they cannot be analyzed by the MCI because their hydrogen lines are unavailable, and we describe them only qualitatively. The system at $z_{\mathrm{abs}}=1.7365$ (separated by $7000 \mathrm{~km} \mathrm{~s}^{-1}$ from the QSO) shows also a clear $\mathrm{N} \mathrm{V}$ doublet (Ly $\alpha$ profile is blended). The apparent ratio $\mathrm{CIV} / \mathrm{N} \mathrm{V}$ is very much like that in the subsystem $A$ at $z_{\mathrm{abs}}=1.6838$ and the $z_{\mathrm{abs}}=1.7365$ system probably has similar physical parameters. The system at $z_{\text {abs }}=1.4978$ $\left(\Delta v=30000 \mathrm{~km} \mathrm{~s}^{-1}\right)$ exhibits a strong and complex Si IV absorption and a weak $\mathrm{NV}$ doublet as well as C II $\lambda 1334$ ( $\mathrm{L} \alpha$ is out of range), and in these features resembles the sub-system $B$ at $z_{\mathrm{abs}}=1.6838$. 


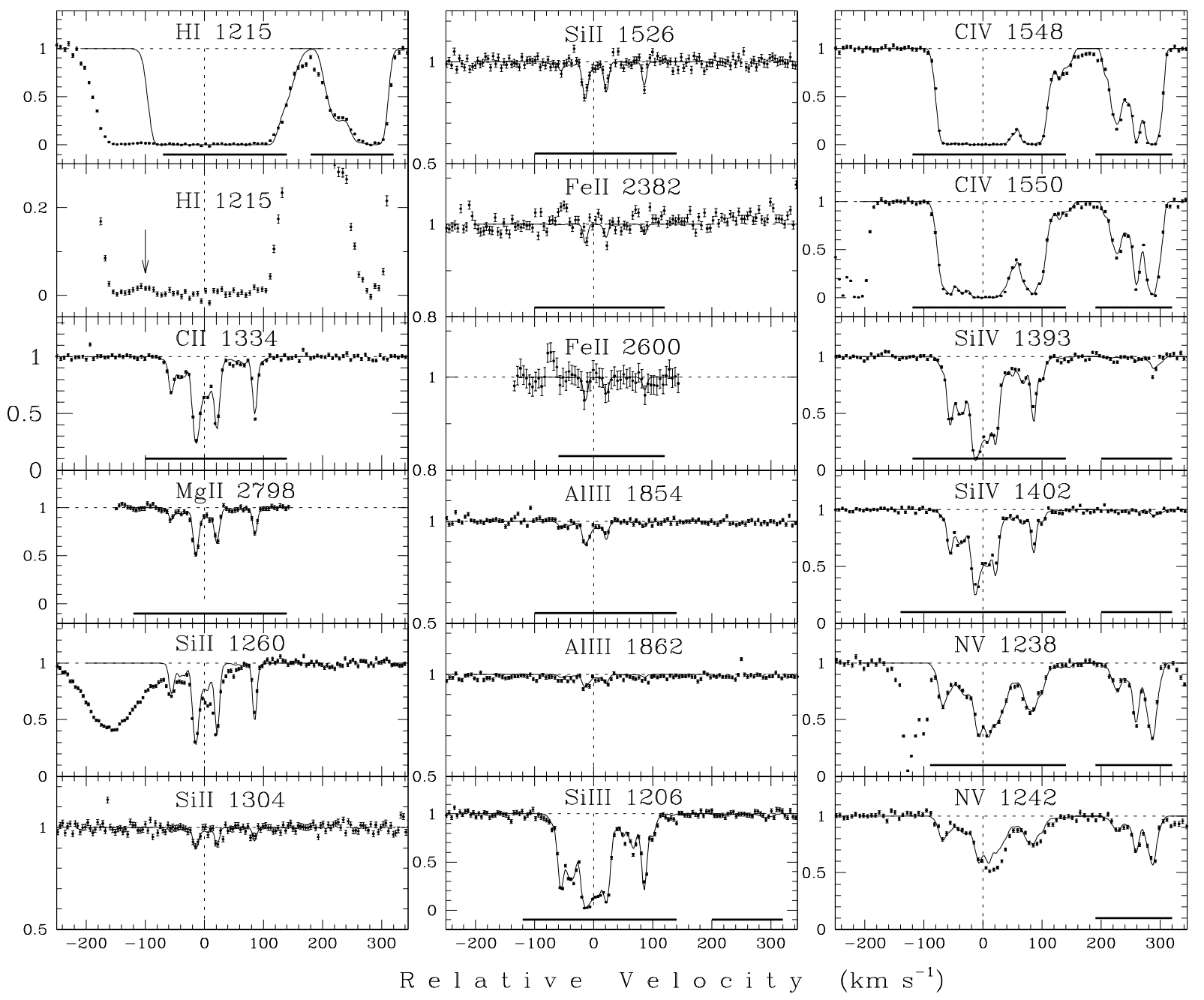

Fig. 5. Same as Fig. 1 but for the $z_{\text {abs }}=1.7103$ system. The zero radial velocity is fixed at $z=1.71027$. The corresponding physical parameters are listed in Table 3, Cols. 3, 4. The arrow in the Ly $\alpha$ panel indicates pixels with non-zero intensities.

\subsection{The distance from the light source}

The oversolar metallicity obtained in the absorbers described above place them in a class of associated systems. Furthermore, their extremely large radial velocities imply that they originate in gas ejected from the circumnuclear region of the QSO/host galaxy. This is also supported by the relatively strong emission flux in Fe II and Fe III lines and at the same time by the Fe II absorptions in the associated systems. Note that Fe II lines in absorbers with $N(\mathrm{HI}) \lesssim 10^{16} \mathrm{~cm}^{-2}$ are extremely rare; probably this is the first such detection.

The distance between the absorbing cloud and the light source can be estimated from the photoionization model and the column density ratios of $\mathrm{C} \mathrm{II} / \mathrm{C}$ II or $\mathrm{Si} \mathrm{II}^{*} / \mathrm{Si}$ II. For an ion in the interstellar (intergalactic) medium, the ratio of excited to ground-state population is equal to the ratio of the collisional excitation rate $Q_{1 \rightarrow 2}$ to the spontaneous transition probability $A_{2 \rightarrow 1}$ (Bahcall \& Wolf 1968):

$\frac{n_{2}}{n_{1}}=\frac{Q_{1 \rightarrow 2}}{A_{2 \rightarrow 1}}$.

The corresponding atomic data for $\mathrm{C} \mathrm{II}^{*}$ and $\mathrm{Si} \mathrm{II}^{*}$ are the following: $A_{2 \rightarrow 1}=2.291 \times 10^{-6} \mathrm{~s}^{-1}$, the excitation rate by collisions with electrons at $T_{\text {kin }}=10^{4} \mathrm{~K} q_{1 \rightarrow 2}^{\mathrm{e}} \simeq 1 \times 10^{-7}$ $\mathrm{cm}^{3} \mathrm{~s}^{-1}$ for $\mathrm{C} \mathrm{II}^{*}$, and $A_{2 \rightarrow 1}=2.17 \times 10^{-4}{ }^{2} \mathrm{~s}^{-1}, q_{1 \rightarrow 2}^{\mathrm{e}} \simeq$ $2 \times 10^{-7} \mathrm{~cm}^{3} \mathrm{~s}^{-1}$ for Si II* (Silva \& Viegas 2002). Since collisions with other particles have much lower excitation rates, we put $Q_{1 \rightarrow 2}=q_{1 \rightarrow 2}^{\mathrm{e}} n_{\mathrm{e}}$.

We do not detect $\mathrm{Si}_{\text {II* }}$ or C II* lines in the associated systems, therefore clear "continuum windows" at the expected positions of $\mathrm{C}$ II $^{*} \lambda 1335.7$ and $\mathrm{Si}$ II $^{*} \lambda 1264.7$ were used to set upper limits on the column densities of $\mathrm{C}$ II $^{*}$ and Si II* (Table 3 ). For the $3 \sigma$ upper limits on $N\left(\mathrm{Si} \mathrm{II}^{*}\right)$ and $N\left(\mathrm{C} \mathrm{II}^{*}\right)$, Eq. (1) provides $n_{\mathrm{e}}<3 \mathrm{~cm}^{-3}$ in the $z_{\mathrm{abs}}=1.78$, and $1.71(A)$ systems, and $n_{\mathrm{e}}<15 \mathrm{~cm}^{-3}$ in the $z_{\mathrm{abs}}=1.68(B)$ system. Since the degree of ionization in these systems is high $\left(n_{\mathrm{H}^{+}} / n_{\mathrm{H}} \gg 1\right)$, the upper limits on the total gas density $n_{\mathrm{H}}$ are the same, i.e., $n_{\mathrm{H}}<3 \mathrm{~cm}^{-3}$ and $n_{\mathrm{H}}<15 \mathrm{~cm}^{-3}$, respectively (the contribution of the ionized helium is ignored since it has a small effect).

To estimate the distance, the QSO continuum luminosity at the Lyman limit $\mathcal{L}_{v_{\mathrm{c}}}$ must be known. Absolute spectrophotometry is not available for HE 0141-3932. Therefore we estimated the intrinsic luminosity $\mathcal{L}_{v_{\mathrm{c}}}$ from $(i)$ the comparison of the QSO $B$ magnitude (assuming the QSO spectral energy distribution is a MF-type, i.e., $\alpha=0.5$ in the range $912 \AA<\lambda<1600 \AA$ ) with 


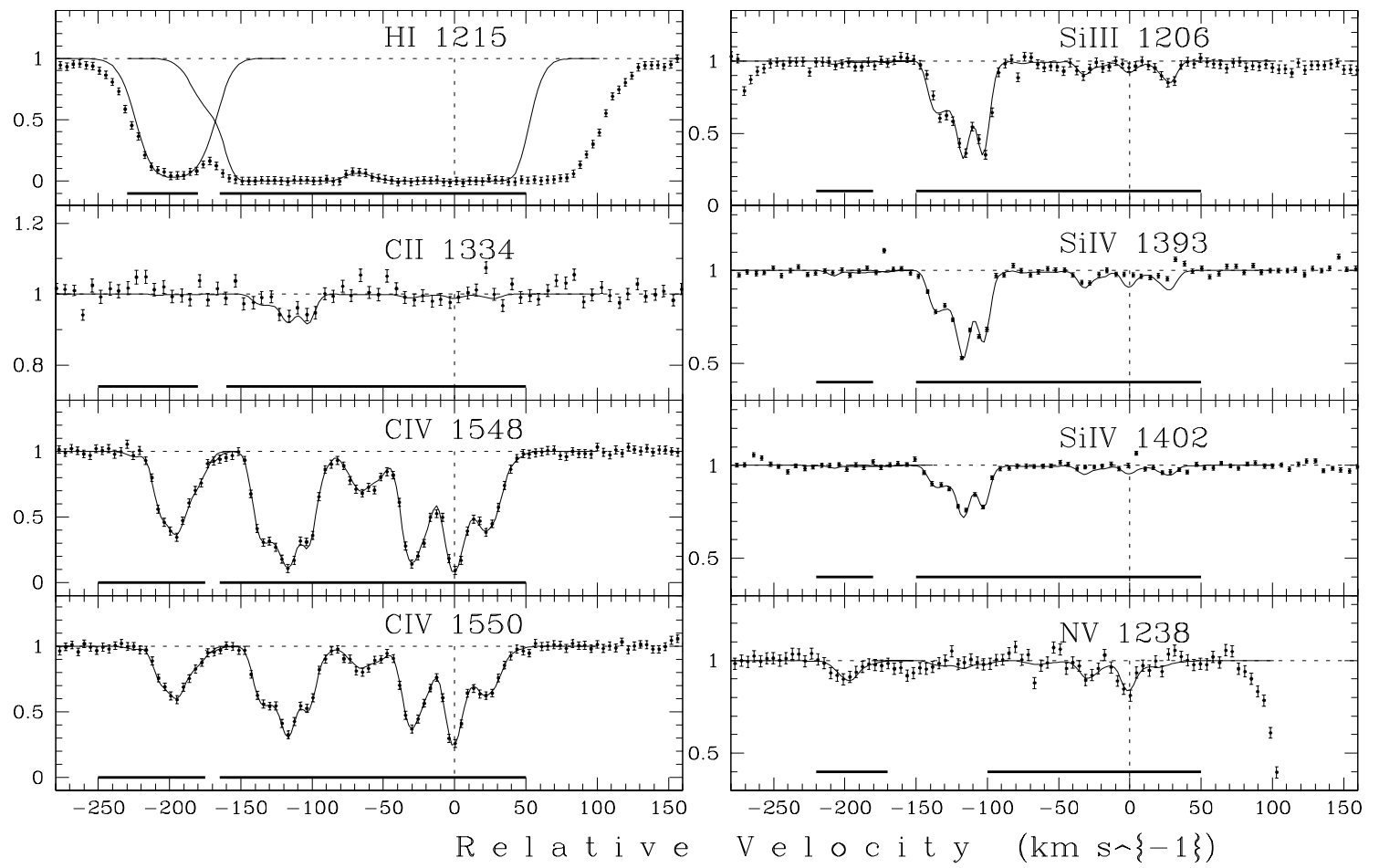

Fig. 6. Same as Fig. 1 but for the $z_{\text {abs }}=1.6838$ system. The zero radial velocity is fixed at $z=1.68377$. The corresponding physical parameters are listed in Table 3, Cols. 5, 6.

the specific flux of a star having $m_{B}=0.0$ outside the Earth's atmosphere $\left(4.4 \times 10^{-20} \mathrm{erg} \mathrm{s}^{-1} \mathrm{~cm}^{-2} \mathrm{~Hz}^{-1}\right)$ and from (ii) the empirical formula given by Tytler (1987, Eq. (17)) which is obtained from a fit to $\left(f_{v}, m_{v}\right)$ data on over 60 QSOs with a wide range of redshifts. The observed $B$ magnitude of 16.09 (corrected for extinction) translates to the flux $f_{v}(4400 \AA)=$ $1.6 \times 10^{-26} \mathrm{erg} \mathrm{s}^{-1} \mathrm{~cm}^{-2} \mathrm{~Hz}^{-1}$ which in turn leads to the apparent luminosity near the Lyman limit $\mathcal{L}_{v_{\mathrm{c}}} \sim 8 \times 10^{31} \mathrm{erg} \mathrm{s}^{-1} \mathrm{~Hz}^{-1}$ $\left(H_{0}=71 \mathrm{~km} \mathrm{~s}^{-1} \mathrm{Mpc}^{-1}, \Omega_{\mathrm{m}}=0.3\right.$, and $\Omega_{\Lambda}=0.7$ are used to calculate the luminosity distance of $13 \mathrm{Gpc}$ ). The second method gives $\mathcal{L}_{v_{\mathrm{c}}} \sim 5 \times 10^{31} \mathrm{erg} \mathrm{s}^{-1} \mathrm{~Hz}^{-1}$ which shows that both values are in reasonable agreement. In the following we use the second one since it is based on the observational data.

Given the upper limits on $n_{\mathrm{H}}$, the distance from the QSO to the absorbing cloud can be calculated from the estimated ionization parameter $U$ which is defined as

$U=\frac{Q\left(\mathrm{H}^{0}\right)}{4 \pi r^{2} c n_{\mathrm{H}}}=\frac{n_{\mathrm{ph}}}{n_{\mathrm{H}}}$,

where

$Q\left(\mathrm{H}^{0}\right)=\int_{v_{\mathrm{c}}}^{\infty} \frac{\mathcal{L}_{v}}{h v} \mathrm{~d} v$

is the number of hydrogen ionizing photons emitted per unit time by the central source, $c$ is the speed of light, $v_{\mathrm{c}}$ is the frequency of the Lyman continuum edge, and $n_{\mathrm{ph}}$ the corresponding density of ionizing photons.

With the estimated Lyman continuum luminosity $\mathcal{L}_{v_{\mathrm{c}}} \sim 5 \times$ $10^{31} \mathrm{erg} \mathrm{s}^{-1} \mathrm{~Hz}^{-1}$, one finds $Q\left(\mathrm{H}^{0}\right) \sim 7.5 \times 10^{57}$ photons s$^{-1}$, assuming $\mathcal{L}_{v}=\mathcal{L}_{v_{\mathrm{c}}}\left(v / v_{\mathrm{c}}\right)^{-\alpha}$, and $\alpha=1$ in the range $v>v_{\mathrm{c}}$. A substitution of the numerical values in Eq. (2) provides $r_{1.68}>$ $100 \mathrm{kpc}, r_{1.71}>280 \mathrm{kpc}$, and $r_{1.78}>450 \mathrm{kpc}$.

\section{Discussion and conclusions}

The characteristics of both the broad emission lines and associated absorptions of HE 0141-3932 can be best explained by the almost pole-on view of this quasar. The weakness of emission lines is then due to dilution by direct radiation from the accretion disk, whereas the velocity shifts of the associated systems and their distances from the light source can be caused by the entrainment into the large-scale outflowing jet. However, powerful jets propagating to distances of $\sim 0.5 \mathrm{Mpc}$ imply the existence of significant radio radiation, but HE 0141-3932 is a radio-quiet QSO. An upper limit to its radio flux of $1 \mathrm{mJy}$ translates into the intrinsic radio luminosity $\mathcal{L}_{v}<7 \times 10^{31} \mathrm{erg} \mathrm{s}^{-1} \mathrm{~Hz}^{-1}$. This is a radio power of a typical FR I source. Jets in the FR I-type structures are known to be subsonic or slightly oversonic with velocities $1000-10000 \mathrm{~km} \mathrm{~s}^{-1}$, heavier than and isobaric with the external medium (e.g., Hughes 1991). These characteristics are in line with the suggestion that the observed absorptions originate in the entrained clouds. It is also known that FR I jets do not show an extended radio emission. This means that radio observations of such jets, especially when they are seen at a small angle to the line of sight, need a high spatial resolution and high sensitivity. Nevertheless, the calculated distances of several hundreds of kiloparsecs seem to be $4-5$ times overestimated since the typical jet length of the FR I object is below $100 \mathrm{kpc}$. We cannot explain the source of this discrepancy. It should be noted, however, that distances of hundreds of kiloparsecs for the associated systems are not exceptional (see, e.g., Morris et al. 1986; Tripp et al. 1996; D’Odorico et al. 2004).

The second fact that is hard to explain is the large velocity excess between low- and high-ionization emission lines. 
According to a model of the quasar atmosphere (e.g., Elvis 2004), low-ionization emission lines ( $\mathrm{H} \alpha$, O I, Fe II, Mg II, $\mathrm{C}$ III]) come from the outer region of the accretion disk which is well shielded from the central source radiation and optically thick for Ly $\alpha$. Ly $\alpha$ and high-ionization emission lines (C IV, $\mathrm{Si}$ IV, N V) are formed in a cool phase of the wind arising from the inner parts of the accretion disk. The emission at $3400 \AA$ observed in HE 0141-3932 cannot be interpreted unambiguously, but if it is (even partially) due to Ly $\alpha$ at $z=1.80$, then its redshift and strength is inconsistent with this scenario since $\mathrm{C}$ IV and Si IV are seen at $z=1.75$. The same situation (i.e., $z_{\mathrm{Ly} \alpha}>z_{\text {CIV }}$ ) is observed in PG $1407+265$. Apparently, a complex geometrical model of the broad emission line region is necessary to explain the observations.

As already mentioned, the relativistic jet beamed toward us was discovered in the bright $(B=15.7)$ and radio-quiet PG 1407+265 (Blundell et al. 2003). There are indications that HE 0141-3932 may also have a similar small-scale relativistic jet. The Lyman limit luminosity of HE 0141-3932 is $\mathcal{L}_{v_{\mathrm{c}}}=5 \times 10^{31} \mathrm{erg} \mathrm{s}^{-1} \mathrm{~Hz}^{-1}$, whereas its radio luminosity is less than $7 \times 10^{31} \mathrm{erg} \mathrm{s}^{-1} \mathrm{~Hz}^{-1}$. This implies either an unusually flat radiation continuum (typically for QSOs is $\mathcal{L}_{v} \propto v^{-0.5}$ at $v<$ $10^{13} \mathrm{~Hz}$ and $\propto v^{-1.5}$ above), or that the apparent optical luminosity is Doppler-boosted due to the relativistic motion of the light source. Another argument in favor of boosting comes from the metallicity estimations. HE $0141-3932$ is a bright source. It is supposed that the QSO luminosity is determined by the accretion rate which requires a large amount of circumnuclear gas. This, in turn, supposes a large mass of the contributing stellar population and, hence, a high metal enrichment of the accreting gas. For reference, all QSOs with luminosities above $5 \times 10^{31} \mathrm{erg} \mathrm{s}^{-1} \mathrm{~Hz}^{-1}$ in the sample of Dietrich et al. (2003) have metallicities $Z>3 Z_{\odot}$ with the mean value of $4-5 Z_{\odot}$. The metallicity of $4-5 Z_{\odot}$ has been also measured in the associated system of a very bright QSO HE 0515-4414 (Levshakov et al. 2003b). Gas in HE 0141-3932 has metallicity $Z \approx 1-2 Z_{\odot}$, which supposes a relatively low stellar population involved in the enrichment, low accretion rate and, hence, low luminosity. Boosting can be caused by a relativistic jet seen at a small viewing angle. Taking into account the measured equivalent widths of the emission lines, we may assume that the luminosity is amplified by $\sim 10$ times, i.e. the intrinsic luminosity of HE 0141-3932 might be only $\mathcal{L}_{v_{\mathrm{c}}} \sim 5 \times 10^{30} \mathrm{erg} \mathrm{s}^{-1} \mathrm{~Hz}^{-1}$.

Another issue of interest is the relative abundances obtained for the absorbing gas. The analyzed absorption systems show high iron content, $[\mathrm{Fe} / \mathrm{C}]=0.15 \pm 0.1,[\mathrm{Fe} / \mathrm{Mg}]=0.0 \pm 0.1$ $\left(z_{\text {abs }}=1.78\right)$, but at the same time nitrogen is strongly underabundant, $[\mathrm{N} / \mathrm{C}] \lesssim-0.5\left(z_{\mathrm{abs}}=1.68\right)$. Although these values were estimated in different absorption systems they are representative for the bulk of circumnuclear gas for the following reason. The mass of the stellar population involved in the enrichment of a quasar's circumnuclear region is $>10^{4} M_{\odot}$ (Baldwin et al. 2003a) and hence large metallicity gradients and sharp discontinuities due to enrichment by only a few stars are unlikely. In the Hamann \& Ferland $(1993,1999)$ models of QSO chemical evolution, solar metallicity is reached after $\gtrsim 0.2 \mathrm{Gyr}$ and is characterized by a relative overabundance of nitrogen, $[\mathrm{N} / \mathrm{C}] \gtrsim 0$, and an underabundance of iron,
$[\mathrm{Fe} / \mathrm{C}]<0$. Due to the delay of $1 \mathrm{Gyr}$ in $\mathrm{Fe}$ enrichment expected from the longer evolution of SNe Ia which are the main sources of iron, the emission line ratios $\mathrm{Fe} \mathrm{II} / \mathrm{C}$ IV and $\mathrm{Fe}$ II/Mg II were proposed as a clock to constrain the QSO ages. However, in these models, large values of $[\mathrm{Fe} / \mathrm{C}]$ and $[\mathrm{Fe} / \mathrm{Mg}]$ are always associated with a considerable overabundance of nitrogen, $[\mathrm{N} / \mathrm{C}]>0.3$. We do not observe such behavior in our systems and, hence, cannot confirm this "iron clock". This is in line with the result of Matteucci \& Recchi (2001), who showed that the time scale for enrichment by SNe Ia is not unique but a strong function of the adopted stellar lifetimes, initial mass function and star formation rate and can vary by more than an order of magnitude.

Acknowledgements. The work of S.A.L. and I.I.A. is supported by the RFBR grant No. 03-02-17522 and by the RLSS grant 1115.2003.2. C.F. is supported by the Verbundforschung of the BMBF/DLR grant No. 50 OR 9911 1. S.L. acknowledges support from the Chilean Centro de Astrofísica FONDAP No. 15010003, and from FONDECYT grant $N^{\circ} 1030491$.

\section{References}

Allende Prieto, C., Lambert, D. L., \& Asplund, M. 2002, ApJ, 573, L137

Bahcall, J. N., \& Wolf, R. A. 1968, ApJ, 152, 701

Baldwin, J. A., Ferland, G. J., Korista, K. T., et al. 2003a, ApJ, 582, 590

Baldwin, J. A., Hamann, F., Korista, K. T., et al. 2003b, ApJ, 583, 649

Blundell, K. M., Beasley, A. J., \& Bicknell, G. V. 2003, ApJ, 591, L103

Box, G. E. P., Hunter, W. G., \& Hunter. J. S. 1978, Statistics for Experimenters: An Introduction to Design, Data Analysis, and Model Building (NY: Wiley \& Sons)

Dietrich, M., Hamann, F., Shields, J. C., et al. 2003, ApJ, 589, 722

D’Odorico, V., Cristiani, S., Romano, D., Granato, G. L., \& Danese, L. 2004, MNRAS, 351, 976

Elvis, M. 2004, in AGN Physics with the Sloan Digital Sky Survey, ed. G. T. Richards, \& P. B. Hall, in press [arXiv: astro-ph/0311436]

Espey, B. R., Carswell, R. F., Bailey, J. A., Smith, M. G., \& Ward, M. J. 1989, ApJ, 342, 666

Fan, X., Strauss, M. A., Schneider, D. P., et al. 2003, AJ, 125, 1649

Fan, X., Strauss, M. A., Gunn, J. E., et al. 1999, ApJ, 526, L57

Ferland, G. J. 1997, A Brief Introduction to Cloudy (Internal Rep., Lexington: Univ. Kentucky)

Francis, P. J. 1993, ApJ, 405, 119

Haardt, F., \& Madau, P. 1996, ApJ, 461, 20 [HM]

Hall, B. P., Snedden, S. A., Niederste-Ostholt, M., et al. 2004, AJ, in press [arXiv: astro-ph/0405239]

Hamann, F., \& Ferland, G. 1999, ARA\&A, 37, 487

Holweger, H. 2001, in Solar and Galactic Composition, ed. R. F. Wimmer-Schweingruber, AIP Conf. Proc., 598, 23

Johnson, N. L., \& Leone, F. C. 1977, Statistics and Experimental Design, vol. II (NY: Wiley \& Sons)

Klein, R. I., McKee, C. F., \& Colella, P. 1994, ApJ, 420, 213

Klein, R. I., Budil, K. S., Perry, T. S., \& Bach, D. P. 2003, ApJ, 583, 245

Korista, K., Baldwin, J., Ferland, G., \& Verner, D. 1997, ApJS, 108, 401 
Hughes, P. A. 1991, Beams and Jets in Astrophysics (Cambridge, UK: University Press)

Leighly, K. M., Halpern, J. P., \& Jenkins, E. B. 2004, in AGN Physics with the Sloan Digital Sky Survey, ed. G. T. Richards, \& P. B. Hall, in press [arXiv: astro-ph/0402535]

Levshakov, S. A., D'Odorico, S., Agafonova, I. I., \& Dessauges-Zavadsky, M. 2004, A\&A, 413, 827

Levshakov, S. A., Agafonova, I. I., D’Odorico, S., Wolfe, A. M., \& Dessauges-Zavadsky, M. 2003a, ApJ, 582, 596

Levshakov, S. A., Agafonova, I. I., Reimers, D., \& Baade, R. 2003b, A\&A, 404, 449

Levshakov, S. A., Agafonova, I. I., Centurión, M., \& Mazets, I. E. 2002, A\&A, 383, 813
Levshakov, S. A., Agafonova, I. I., \& Kegel, W. H. 2000, A\&A, 360, 833

Mathews, W. G., \& Ferland, G. J. 1987, ApJ, 323, 456 [MF]

McDowell, J. C., Canizares, C., Elvis, M., et al. 1995, ApJ, 450, 585

Morris, S. L., Weymann, R. J., Foltz, C. B., et al. 1986, ApJ, 310, 40

Morton, D. C. 2003, ApJS, 149, 205

Silva, A. I., \& Viegas, S. M. 2002, MNRAS, 329, 135

Srianand, R., \& Petitjean, P. 2001, A\&A, 373, 816

Tripp, T. M., Lu, L., \& Savage, B. D. 1996, ApJS, 102, 239

Tytler, D. 1987, ApJ, 321, 69

Urry, C. M., \& Padovani, P. 1995, PASP, 107, 803

Wisotzki, L., Christlieb, N., Bade, N., et al. 2000, A\&A, 358, 77 
D. Reimers et al.: HE 0141-3932: A bright QSO with an unusual emission line spectrum and associated absorption, Online Material p 1

\section{Online Material}




\section{Appendix A: Evaluation of the spectral shape of the local UV background}

In general, the ionizing UV background is produced by the attenuated UV flux of QSOs and/or other sources (like young galaxies) and by the recombination radiation from intergalactic gas clouds. To estimate the shape of the UV continuum from the observed profiles of ions in the metal systems we use the response function methodology from the theory of experimental design (see, e.g., Box et al. 1978, Chap. 15).

At first, the shape of the UV continuum in the range $E>1$ Ryd is to be parameterized by means of $k$ variables (factors). For instance, the AGN-type spectrum can be described by a broken power-law defined by the following factors (see Fig. 4): $f_{1}$ - the power law index (slope) in the range 1 Ryd $<E<f_{2}$ Ryd; $f_{2}$ - the coordinate of the first fracture; $f_{3}$ the slope between $f_{2}$ and the point of the second fracture $f_{4} ; f_{5}$ the value of the break after $f_{4}$ (decimal logarithm of the intensities ratio) with the slope $f_{6} ; f_{7}$ the slope in the high $\mathrm{UV}$ range. Formally we fix the starting point of the X-ray break at $E=100$ Ryd and the slope after it at -1.5 since this spectral range affects very weakly the fractional ionizations of ions we are interested in. In this factor space, the Mathews-Ferland (MF) spectrum is represented by a point with the coordinates $\{-0.5,0.24,-1.0,0.61,-2.5,-3.0,-0.7\}$.

At the start of the procedure we select a basic UV spectrum (e.g. power law, HM, or MF) and carry out the MCI calculations using the fractional ionizations $\Upsilon_{\mathrm{a}, i}{ }^{1}$ produced by this background.

If the trial spectrum turns out to be inconsistent with the observed line intensities, we assign its parameters to a "null point" in the factor space and vary the factors around this null point according to the chosen set of treatments ${ }^{2}$. Thus, for each treatment we obtain a new trial UV spectrum.

The fitness of a particular spectrum is measured by the value of the response function $\mathcal{R}$ which is defined individually for each absorption system in such a way as to ensure the self-consistent description of all absorption lines detected in the system. The best UV spectrum is that which provides a maximum value of $\mathcal{R}$ within the constraints $\chi^{2} \leqslant 1$ (per degree of freedom) for each individual line. The information needed to construct the response function is obtained in several test runs with the "null spectrum".

The following example illustrates this step of the procedure. Let us assume that an absorption system exhibits lines of Si II, Si IV and C II, C IV. Trial calculations with a basic UV continuum show that this continuum overpredicts the intensity of Si II and underpredicts Si IV whereas the behavior of carbon lines is opposite - underpredicted C II and overpre-

\footnotetext{
${ }^{1}$ If we define $n_{\mathrm{a}, i}$ as the total ion number density of element "a" in the $i$ th ionization stage $\left(n_{\mathrm{a}}=\sum_{i} n_{\mathrm{a}, i}\right)$, then $\Upsilon_{\mathrm{a}, i}=n_{\mathrm{a}, i} / n_{\mathrm{a}}$ is the fractional ionization of ion $\{\mathrm{a}, i\}$. For a given metallicity $Z_{\mathrm{a}}, n_{\mathrm{a}, i}=$ $\Upsilon_{\mathrm{a}, i} Z_{\mathrm{a}} n_{\mathrm{H}}$.

2 The treatment is a particular combination of values of the factors involved in an experiment. The set of treatments is called the "experimental plan". Experimental plans for any number of factors can be found in corresponding reference books (see, e.g., Johnson \& Leone 1977).
}

dicted C IV. In this case it is conceivable to search for such a background that will produce a maximum value of the product $\mathrm{Si}$ IV/Si II and C II/C IV. The line intensities (and column densities) are proportional to the fractional ionizations and we can calculate the response function simply as

$\mathcal{R}=\frac{\Upsilon_{\mathrm{SiIV}}}{\Upsilon_{\mathrm{Si} \text { II }}} \times \frac{\Upsilon_{\mathrm{CII}}}{\Upsilon_{\mathrm{CIV}}}$.

Fractional ionizations for each trial UV background are taken from CLOUDY. Apart from the ionizing spectrum, further input parameters for CLOUDY are the ionization parameter $U$, metallicity and element ratios (e.g. $[\mathrm{Si} / \mathrm{C}],[\mathrm{O} / \mathrm{C}]$ ) which are estimated in test runs.

After the response function has been evaluated at each point of the experimental plan, factor weights (influences) $\left\{\alpha_{i}\right\}_{i=1}^{k}$ can be estimated. For this purpose a standard polynomial model is used:

$\mathcal{R}=\sum_{i=1}^{k} \alpha_{i} \hat{f}_{i}+\beta$,

where $\hat{f}_{i}$ is the normalized and centered value of $i$ th factor, $\hat{f_{i}}=\left(f_{i}-f_{0, i}\right) / \sigma_{i}, \sigma_{i}-$ variability level of $i$ th factor. Note that this form of $\mathcal{R}$ defines a response surface in $k$-dimensional factor space. The coefficients $\alpha_{i}$ and $\beta$ are estimated from $n$ $(n \geq k+1)$ values of $\mathcal{R}$ computed at the plan points. The input of the nonlinear term can be deduced through the comparison of the estimate of $\hat{\beta}$ and the value of $\mathcal{R}_{0}$ at the "null point", i.e. when $f_{i}=f_{0, i}$. The statistical significance of $\left(\mathcal{R}_{0}-\hat{\beta}\right)$ points to non-negligible factor interactions. Then additional points (treatments) should be added to the experimental plan to estimate the second-order terms. The optimal spectrum is obtained by moving the factor values in the direction normal to the response surface. For the absorption systems described in the present paper a linear model turned out to be sufficient.

After the first iteration of the spectral shape adjustment is completed, we repeat the MCI calculations with the designed UV background and, if necessary, go back to the adjustment procedure until a satisfactory result (i.e. normalized $\chi^{2} \lesssim 1$ for all profiles included in the analysis) is achieved. 